Pacific Journal of Mathematics

HOMOLOGICAL DIMENSION OF ORE-EXTENSIONS 


\title{
HOMOLOGICAL DIMENSION OF ORE-EXTENSIONS
}

\author{
N. S. Gopalakrishnan and R. SRIDHaRan
}

Let $S$ be a ring with unit element and let $R=S\{x, d\}$ be the Ore-extension of $S$ with respect to a derivation $d$ of $S$. Our object in this paper is to show that $l . g l . \operatorname{dim} R=1+$ l. gl. $\operatorname{dim} S$, if $S$ is a commutative Noetherian ring and $d$ is suitably restricted.

It was shown in [3] that l. gl. $\operatorname{dim} R \leqq 1+1$. gl. $\operatorname{dim} S$. While equality does not hold in general, we show that it does under suitable conditions (Theorem 2, §5).

This is achieved in three steps. The first is to show that for any ring $S$, any $R$-module $M$ and an $S$-projective resolution for $M$, there exists an $R$-projective resolution of $M$ which "lifts" the given resolution (Theorem 1, §3). The next step is to use this resolution to prove Theorem 2 in the special case in which $S$ is a local ring (Proposition $1, \S 4)$. The final step consists in deducing Theorem 2 by the method of localisation.

The authors would like to express their thanks to M. P. Murthy and A. Roy for their kind help during the preparation of this paper.

2. Preliminaries on Ore-extensions. Let $S$ be a ring with unit element (denoted by 1), which is not necessarily commutative, and let $d$ be a derivation of $S$ into itself. Let $S\{x, d\}$ denote the Ore-extension of $S$ with respect to $d$ (see [5]). We recall that $R=S\{x, d\}$ is the ring generated by an indeterminate $x$ over $S$ with the relations $x s-s x=d s$ for every $s \in S$. We identify $S$ with a subring of $R$. We collect here some properties of $R$ which will be used in the later sections.

(2.1) For any ring $S^{\prime}$, a ring homomorphism $\varphi: S \rightarrow S^{\prime}$ and an element $\alpha \in S^{\prime}$, with the property $\alpha \varphi(s)-\varphi(s) \alpha=\varphi(d s)$, there exists a unique ring homomorphism $\bar{\varphi}: R \rightarrow S^{\prime}$ such that $\bar{\varphi}(x)=\alpha$ and $\bar{\varphi} \mid S=\varphi$. (In fact $R$ can be characterised by this property).

The proof is straightforward.

(2.2) Let $S_{1}, S_{2}$ be rings with derivations $d_{1}, d_{2}$ respectively and let $\varphi: S_{1} \rightarrow S_{2}$ be a ring homomorphism such that $d_{2} \circ \varphi=\varphi \circ d_{1}$. Then there exists a ring homomorphism $\bar{\varphi}: R_{1} \rightarrow R_{2}$ such that $\bar{\varphi} \mid S_{1}=\varphi$.

Proof. This follows from (2.1) by taking $S^{\prime}=R_{2}$ and $\alpha=x \in R_{2}$.

(2.3) A left $S$-module $M$ can be converted to a left- $R$-module if

Received January 25, 1965. 
and only if there exists an $f \in \operatorname{Hom}_{\mathrm{Z}}(M, M)$ such that $f(s . m)-s . f(m)=$ $d s . m$, for every $s \in S, m \in M$.

Proof. If $M$ is an $R$-module we may take $f \in \operatorname{Hom}_{\mathrm{Z}}(M, M)$ defined by $f(m)=x . m$. The converse follows from (2.1) by taking

$$
S^{\prime}=\operatorname{Hom}_{\mathrm{Z}}(M, M), \alpha=f \quad \text { and } \quad \varphi: S \rightarrow S^{\prime}
$$

to be the mapping which defines the $S$-module structure on $M$.

(2.4) If $M$ is a projective left $S$-module, then $M$ can be converted into a left $R$-module.

Proof. We first remark that $S$ can be considered as a left $R$-module. In fact, with the notation of (2.3) we choose $f=d \in \operatorname{Hom}_{\mathrm{Z}}(S, S)$. By a direct sum argument, it is clear that any free left $S$-module can be regarded as an $R$-module. Now let $M$ be any projective left $S$-module and let $M$ be a direct summand of a free $S$-module $F$. Since $F$ is a left $R$-module, there exists an $f \in \operatorname{Hom}_{\mathrm{Z}}(F, F)$ such that $f(s . m)$ s. $f(m)=d s . m ; s \in S, m \in F$. Let $p: F \rightarrow M$ be an $S$-projection of $F$ on $M$. It is easily seen that $g=f \circ p \mid M$ satisfies $g(s . m)-s . g(m)=d s . m$. Hence $M$ can be regarded as an $R$-module.

(2.5) $R$ becomes a filtered ring by setting $F_{p} R=\sum_{0 \leqq i \leqq p} S . x^{i}$. The associated graded ring $E^{\circ}(R)$ of $R$ is isomorphic to $S[x]$, the usual polynomial ring in one variable $x$ over $S$.

Proof. See [3].

3. Lifting of resolutions. Let $M$ be a left $R$-module and let

$$
\cdots \longrightarrow X_{i} \stackrel{d_{i}}{\longrightarrow} X_{i-1} \longrightarrow \cdots \longrightarrow X_{0} \stackrel{\varepsilon}{\longrightarrow} M \longrightarrow 0
$$

be an $S$-projective resolution of $M$. Our aim in this section is to construct an $R$-projective resolution which "lifts" the above resolution.

We first prove the following

Lemma. There exist $f_{i} \in \operatorname{Hom}_{\mathbb{Z}}\left(X_{i}, X_{i}\right)$ such that

(i) $f_{i}(s . \alpha)-s . f_{i}(\alpha)=d s . \alpha$ for $s \in S, \alpha \in X_{i}$;

(ii) $d_{i} \circ f_{i}=f_{i-1} \circ d_{i}, i \geqq 1$, and $\varepsilon \circ f_{0}=f \circ \varepsilon$, where $f \in \operatorname{Hom}_{\mathrm{Z}}(M, M)$ is the mapping given by $f(m)=x . m$.

Proof. Since $X_{0}$ is $S$-projective, it follows from (2.4) and (2.3) that there exists an $f_{0}^{\prime} \in \operatorname{Hom}_{\mathbb{Z}}\left(X_{0}, X_{0}\right)$ such that $f_{0}^{\prime}(s \alpha)-s f_{0}^{\prime}(\alpha)=d s, \alpha$ for $s \in S, \alpha \in X_{0}$. The map $\varepsilon \circ f_{0}^{\prime}-f \circ \varepsilon: X_{0} \rightarrow M$ is easily verified to be $S$-linear. Since $X_{0}$ is $S$-projective there exists an $f_{0}^{\prime \prime} \in \operatorname{Hom}_{\mathbb{S}}\left(X_{0}, X_{0}\right)$ 
such that $\varepsilon \circ f_{0}^{\prime}-f \circ \varepsilon=\varepsilon \circ f_{0}^{\prime \prime}$. We choose $f_{0}=f_{0}^{\prime}-f_{0}^{\prime \prime}$. Then (i) and (ii) are verified for $i=0$.

Assume inductively that $f_{j} \quad 0 \leqq j \leqq i-1$ have already been defined satisfying (i) and (ii). Since $X_{i}$ is $S$-projective, there exists $f_{i}^{\prime} \in \operatorname{Hom}_{\mathrm{Z}}\left(X_{i}, X_{i}\right)$ such that $f_{i}^{\prime}(s \alpha)-s f_{i}^{\prime}(\alpha)=d s \alpha$ for $s \in S, \alpha \in X_{i}$. The map $d_{i} \circ f_{i}^{\prime}-f_{i-1} \circ d_{i}: X_{i} \rightarrow X_{i-1}$ is easily verified to be $S$-linear. We have, (with the convention $f_{1}=f$ and $d_{0}=\varepsilon$ ),

$$
\begin{aligned}
d_{i-1}\left(d_{i} \circ f_{i}^{\prime}-f_{i-1} \circ d_{i}\right) & =-d_{i-1} \circ f_{i-1} \circ d_{i} \\
& =-f_{i-2} \circ d_{i-1} \circ d_{i} \quad \text { (by induction) } \\
& =0 .
\end{aligned}
$$

Hence the image of $X_{i}$ by $d_{i} \circ f_{i}^{\prime}-f_{i-1} \circ d_{i}$ is contained in the kernel of $d_{i-1}=\operatorname{Im} . d_{i}$. Since $X_{i}$ is $S$-projective, there exists $f_{i}^{\prime \prime} \in \operatorname{Hom}_{\mathrm{S}}\left(X_{i}, X_{i}\right)$ such that $d_{i} \circ f_{i}^{\prime}-f_{i-1} \circ d_{i}=d_{i} \circ f_{i}^{\prime \prime}$. We may choose $f_{i}=f_{i}^{\prime}-f_{i}^{\prime \prime}$ and $f_{i}$ satisfies (i) and (ii). This completes the proof of the lemma.

We set $X_{-1}=0$ and define for $i \geqq 0$

$$
\bar{X}=R \bigotimes_{S} X_{i}+R y \bigotimes_{S} X_{i-1},
$$

where $y$ is a dummy. We set $d_{0}=0$ and define for $i \geqq 1$, the $R$-homomorphism $\bar{d}_{i}: \bar{X}_{i} \rightarrow \bar{X}_{i-1}$ by

$$
\bar{d}_{i}\left(1 \otimes \alpha^{\prime}\right)=1 \otimes d_{i} \alpha, \alpha \in X_{i}
$$

and

$$
\bar{d}_{i}\left(y \otimes \alpha^{\prime}\right)=y \otimes d_{i-1} \alpha^{\prime}+(-1)^{i-1} x \otimes \alpha^{\prime}+(-1)^{i} 1 \otimes f_{i-1}\left(\alpha^{\prime}\right), \alpha^{\prime} \in X_{i-1} .
$$

We define the $R$-homomorphism $\bar{\varepsilon}: \bar{X}_{0}=R \bigotimes_{S} X_{0} \rightarrow M$ by

$$
\bar{\varepsilon}(1 \otimes \alpha)=\varepsilon(\alpha), \alpha \in X_{0} \text {. }
$$

THEOREM 1. The sequence

$$
\cdots \longrightarrow \bar{X}_{i} \stackrel{\bar{d}_{i}}{\longrightarrow} \bar{X}_{i-1} \longrightarrow \cdots \longrightarrow \bar{X}_{0} \stackrel{\bar{\varepsilon}}{\longrightarrow} M \longrightarrow 0
$$

is an $R$-projective resolution of $M$.

Proof. For $\alpha \in X_{1}, \bar{\varepsilon} \circ \bar{d}_{1}(1 \otimes \alpha)=\bar{\varepsilon}\left(1 \otimes d_{1} \alpha\right)=\varepsilon d_{1}(\alpha)=0$, and for

$$
\begin{aligned}
\alpha^{\prime} \in X_{0}, \bar{\varepsilon}^{\circ} \circ \bar{d}_{1}\left(y \otimes \alpha^{\prime}\right) & =\bar{\varepsilon}\left(x \otimes \alpha^{\prime}-1 \otimes f_{0}\left(\alpha^{\prime}\right)\right) \\
& =f \circ \varepsilon\left(\alpha^{\prime}\right)-\varepsilon \circ f_{0}\left(\alpha^{\prime}\right)=0 .
\end{aligned}
$$

For $i \geqq 1$, we have

$$
\bar{d}_{i-1} \circ \bar{d}_{i}(1 \otimes \alpha)=1 \otimes d_{i-1} \circ d_{i} \alpha=0, \alpha \in X_{i},
$$

and 


$$
\begin{aligned}
& \bar{d}_{i-1} \circ \bar{d}_{i}\left(y \otimes \alpha^{\prime}\right) \\
&=\bar{d}_{i-1}\left[y \otimes d_{i-1} \alpha^{\prime}+(-1)^{i-1} x \otimes \alpha^{\prime}+(-1)^{i} 1 \otimes f_{i-1}\left(\alpha^{\prime}\right)\right], \alpha^{\prime} \in X_{i-1} \\
&=y \otimes d_{i-2} \circ d_{i-1}+(-1)^{i-2} x \otimes d_{i-1} \alpha^{\prime}+(-1)^{i-1} 1 \otimes f_{i-2} d_{i-1} \alpha^{\prime} \\
& \quad+(-1)^{i-1} x \otimes d_{i-1} \alpha^{\prime}+(-1)^{i} 1 \otimes d_{i-1} \circ f_{i-1} \alpha^{\prime} \\
&=(-1)^{i} 1 \otimes\left(d_{i-1} \circ f_{i-1}-f_{i-2} \circ d_{i-1}\right),
\end{aligned}
$$

(with the convention that $f_{-1}=0$ )

$$
=0 \text {. }
$$

Thus $(*)$ is a complex of left $R$-modules. To prove that the complex is acyclic, we define a suitable filtration on the complex whose associated graded is acyclic. By a well-known lemma on filtered complexes the acyclicity of $(*)$ follows immediately. For $i \geqq 0$, let

$$
F_{p} \bar{X}_{i}=F_{p} R \bigotimes_{S} X_{i}+F_{p-1} R . y \bigotimes_{S} X_{i-1},
$$

where $\left\{F_{p} R\right\}$ is the filtration on $R$ defined in (2.5). We define

$$
F_{p} M=M \text { for every } p \text {. }
$$

It is easily seen that $\left\{F_{p} \bar{X}_{i}\right\}$ defines a filtration on $\bar{X}_{i}$ and that $\bar{d}_{i}\left(F_{p} \bar{X}_{i}\right) \subset F_{p} \bar{X}_{i-1}$ for $i \geqq 1$ and $\varepsilon\left(F_{p} X_{0}\right) \subset F_{p} M$. We thus get for $p \geqq 0$ the complex

$$
\cdots \longrightarrow E_{p}^{0}\left(\bar{X}_{i}\right) \stackrel{E_{p}^{0} \overline{\left(d_{i}\right)}}{\longrightarrow} E_{p}^{0}\left(\bar{X}_{i-1}\right) \longrightarrow \cdots \longrightarrow E_{p}^{0}\left(\bar{X}_{0}\right) \stackrel{E_{p}^{0}(\bar{\varepsilon})}{\longrightarrow} E_{p}^{0}(M) \longrightarrow 0 .
$$

We note that $E_{p}^{0}(M)=0$ for $p \neq 0$ and $E_{0}^{0}(M)=M$.

Let $S[x]$ denote the polynomial ring in one variable $x$ over $S$. We regard $M$ as an $S[x]$-module by setting $x M=0$. We set $X_{-1}^{\prime}=0$ and define $X_{i}^{\prime}$ for $i \geqq 0$ by

$$
X_{i}^{\prime}=S_{p}[x] \bigotimes_{S} X_{i}+S_{p-1}[x] \cdot y \bigotimes_{S} X_{i-1}
$$

We set $d_{0}^{\prime}=0$ and for $i \geqq 1$ define the left $S[x]$-homomorphism $d_{i}^{\prime}: X_{i}^{\prime} \rightarrow X_{i-1}^{\prime}$ by

$$
\begin{aligned}
d_{i}^{\prime}(1 \otimes \alpha) & =1 \otimes d_{i} \alpha, \alpha \in X_{i}, \\
d_{i}^{\prime}\left(y \otimes \alpha^{\prime}\right) & =y \otimes d_{i-1} \alpha^{\prime}+(-1)^{i-1} x \otimes \alpha^{\prime}, \alpha^{\prime} \in X_{i-1} .
\end{aligned}
$$

We define the $S[x]$-homomorphism $\varepsilon^{\prime}: X_{0}^{\prime} \rightarrow M$ by setting

$$
\varepsilon^{\prime}(1 \otimes \alpha)=\varepsilon(\alpha) \text {. }
$$

It is easily verified $[4, \mathrm{p} .210]$ that $\left(X_{i}^{\prime}, d_{i}^{\prime}\right)$ is a left $S[x]$-projective resolution for $M$.

Let $S_{p}[x]$ be the $p^{t h}$ homogeneous component of the usual gradation of $S[x]$ given by powers of $x$. We introduce a gradation on 
$X_{i}^{\prime}$ by setting

$$
X_{i}^{\prime p}=S_{p}[x] \bigotimes_{S} X_{i}+S_{p-1}[x] y \bigotimes_{S} X_{i-1} .
$$

We take the trivial gradation on $M$ i.e., $M^{p}=0$ for $p>0$ and $M^{0}=M$. It is easily seen that $d_{i}^{\prime}\left(X_{i}^{p}\right) \subset X_{i-1}^{\prime p}$ and $\varepsilon^{\prime}\left(X_{0}^{\prime p}\right) \subset M^{p}$ for every $p$. We thus get for every $p$ an exact sequence

$$
\cdots \longrightarrow X_{i}^{\prime p} \stackrel{d_{i}^{\prime p}}{\longrightarrow} X_{i-1}^{\prime p} \longrightarrow \cdots \longrightarrow X_{0}^{\prime p} \stackrel{\varepsilon^{\prime p}}{\longrightarrow} M^{p} \longrightarrow 0 .
$$

Clearly $E_{p}^{0}\left(\bar{X}_{i}\right) \approx X_{i}^{\prime p}$ and $E_{p}^{0}(M) \approx M^{p}$ for every $p$. Since for any $r \in F_{p-1} R$ and $\alpha^{\prime} \in X_{i-1}$, we have $r \otimes f_{i-1}\left(\alpha^{\prime}\right) \in F_{p-1} \bar{X}_{i-1}$, it follows that $E_{p}^{0}\left(\bar{d}_{i}\right)=d_{i}^{\prime p}$. Since $(* *)$ is exact, it follows that $\left(E_{p}^{0}\left(\bar{X}_{i}\right), E_{p}^{0}\left(\bar{d}_{i}\right)\right)$ is exact and hence $(*)$ is exact. Since $\bar{X}_{i}$ is clearly $R$-projective, the theorem is proved.

4. The case of local rings. Our aim in this section is to prove the following.

Proposition 1. Let $S$ be a (commutative, Noetherian) local ring and let $\mathfrak{M}$ denote its unique maximal ideal. Let $d$ be a derivation of $S$ such that $d(S) \subset \mathfrak{M}$ and let $R=S\{x, d\}$. Then

$$
\text { l.gl. } \operatorname{dim} R=1+\text { gl. } \operatorname{dim} S \text {. }
$$

For proving this proposition, we need the following.

LEMma. Let $S$ be a commutative ring and let $M$ be an $R$-module. Suppose

$$
0 \longrightarrow X_{n} \stackrel{d_{n}}{\longrightarrow} X_{n-1} \stackrel{d_{n-1}}{\longrightarrow} \cdots \longrightarrow X_{0} \longrightarrow M \longrightarrow 0
$$

is an S-projective resolution of $M$. Assume that the following conditions hold.

(1) $X_{n}$ is $S$-free of rank 1.

(2) There exists an $S$-module $N$ with $x N=0$ and $\operatorname{Ext}_{s}^{n}(M, N) \neq$ (0).

Then $h d_{R} M=n+1$.

Proof. Using the complex $(*)$ of Theorem 1 , we find that $h d_{R} M \leqq$ $n+1$. We now compute $\operatorname{Ext}_{R}^{n+1}\left(M, N^{\prime}\right)$ for any $R$-module $N^{\prime}$. We have

$$
\operatorname{Ext}_{R}^{n+1}\left(M, N^{\prime}\right)=\operatorname{Hom}_{S}\left(X_{n}, N^{\prime}\right) / B^{n}
$$

where $B^{n}$ is the set of all $g \in \operatorname{Hom}_{S}\left(X_{n}, N^{\prime}\right)$ such that there exist $g_{1} \in \operatorname{Hom}_{S}\left(X_{n}, N^{\prime}\right)$ and $g_{2} \in \operatorname{Hom}_{S}\left(X_{n-1}, N^{\prime}\right)$ with

$$
g(\alpha)=g_{2}\left(d_{n} \alpha\right)+(-1)^{n-1} x g_{1}(\alpha)+(-1)^{n} g_{1}\left(f_{n}(\alpha)\right)
$$

for any $\alpha \in X_{n}$. 
Let $\beta$ be a free generator of $X_{n}$ as an $S$-module and let $f_{n}(\beta)=$ $s \beta ; s \in S$. If $g \in B^{n}$, we have

$$
g(\beta)=g_{2}\left(d_{n} \beta\right)+(-1)^{n-1}(x-s) g_{1}(\beta) .
$$

Let $\theta$ be the automorphism of $R$ such that $\theta(x)=x+s$ and $\theta \mid S=$ identity. (This exists in view of (2.1)). If we choose $N^{\prime}={ }_{\theta} N$ (i.e., $N$ considered as an $R$-module through $\theta)$, we find $g(\beta)=g_{2}\left(d_{n} \beta\right)$ and hence $g(\alpha)=g_{2}\left(d_{n} \alpha\right)$ for any $\alpha \in X_{n}$. Thus, $B^{n}=B_{1}^{n}=$ $\left\{g \in \operatorname{Hom}_{S}\left(X_{n}, N^{\prime}\right) \mid g(\alpha)=g_{2}\left(d_{n} \alpha\right)\right.$ for some $g_{2} \in \operatorname{Hom}_{S}\left(X_{n-1} N^{\prime}\right)$ for every $\left.\alpha \in X_{n-1}\right\}$. However, using the resolution $\left(X_{i}, d_{i}\right)$ for $M$ to compute Ext, we find $\operatorname{Ext}_{S}^{n}\left(M, N^{\prime}\right) \approx \operatorname{Hom}_{S}\left(X_{n}, N^{\prime}\right) / B_{1}^{n}$. Hence

$$
\begin{aligned}
\operatorname{Ext}_{R}^{n+1}\left(M, N^{\prime}\right) & \approx \operatorname{Ext}_{S}^{n}\left(M, N^{\prime}\right) \\
& \approx \operatorname{Ext}_{S}^{n}(M, N) \neq(0),
\end{aligned}
$$

since $N$ and $N^{\prime}$ are isomorphic as $S$-modules. This proves the lemma.

Proof of proposition. By [2, p. 74, Prop. 2], it follows that gl. $\operatorname{dim} R \geqq$ gl. $\operatorname{dim} S$. Thus, if gl. $\operatorname{dim} S=\infty$, we have gl. $\operatorname{dim} R=\infty$ and the proposition is proved. We therefore assume that $\mathrm{gl} \cdot \operatorname{dim} S=$ $n<\infty$. If $M=S / M$, we have $h d_{S} M=n$. Let

$$
0 \longrightarrow X_{n} \stackrel{d_{n}}{\longrightarrow} X_{n-1} \longrightarrow \cdots \longrightarrow X_{0} \longrightarrow M \longrightarrow 0
$$

be the "Koszul resolution" for $M[1, \mathrm{p} .151]$. Since $X_{n}=E_{n}^{s}\left(y_{1}, \cdots, y_{n}\right)$, where $E_{n}^{S}\left(y_{1}, \cdots, y_{n}\right)$ is the $n$th component of the exterior algebra on $y_{1}, \cdots, y_{n}$ over $S$, condition (i) of the above lemma is satisfied. Since $d(S) \subset \mathfrak{M}$, it is clear that $M$ can be regarded as an $R$-module satisfying $x M=0$ (See (2.3)). Since $\operatorname{Ext}_{S}^{n}(M, M) \neq(0),[1, \mathrm{p} .153]$, condition (2) of the lemma is satisfied with $N=M$. Thus, by the above lemma, we have $h d_{R} M=n+1$. Hence gl. $\operatorname{dim} R \geqq n+1$. Since gl. $\operatorname{dim} R \leqq$ $n+1$ [6, Th. 1 or 3], the proposition is proved.

5. The case of Noetherian rings. In this section, we prove the following

THEOREM 2. Let $S$ be a commutative Noetherian ring and let $d$ be a derivation of $S$ such that any one of the following two conditions is satisfied:

(1) $d(S) \subset$ Radical of $S$,

(2) $d(S)$ generates a proper ideal of $S$ and Krull dim $S_{\mathfrak{M}}$ is the same for all the maximal ideals $\mathfrak{M}$ of $S$.

If $R=S\{x, d\}$, we have

l.gl. $\operatorname{dim} R=1+g l . \operatorname{dim} S$. 
Proof. As in the proof of Proposition 1, we need only prove that l. gl. $\operatorname{dim} R \geqq 1+$ gl. $\operatorname{dim} S$ assuming gl. $\operatorname{dim} S<\infty$. Since gl. $\operatorname{dim} S=$ $\sup _{\mathfrak{M}}$ gl. $\operatorname{dim} S_{\mathfrak{M}}$ where $\mathfrak{M}$ runs over all the maximal ideals of $S$, it is clear that under either of the conditions of the theorem, there exists a maximal ideal $\mathfrak{M}$ such that $\mathrm{gl} \cdot \operatorname{dim} S=\operatorname{gl} \cdot \operatorname{dim} S_{\mathfrak{M}}$ and $d(S) \subset \mathfrak{M}$. The derivation $d$ of $S$ induces a derivation $\bar{d}$ of $S_{\mathfrak{M}}$ if we set

$$
\bar{d}\left(\frac{s}{s^{\prime}}\right)=\frac{d s . s^{\prime}-s . d s^{\prime}}{s^{\prime 2}} ; s, s^{\prime} \in S, s^{\prime} \in \mathfrak{M} \text {. }
$$

It is clear that $\bar{d}\left(S_{\mathfrak{M}}\right) \subset \mathfrak{M} S_{\mathfrak{M}}$. Hence by Proposition $1, \S 4$, we have

$$
\text { l. gl. } \begin{aligned}
\operatorname{dim} S_{\mathfrak{M}}\{x, \bar{d}\} & =1+\text { gl. } \operatorname{dim} S_{\mathfrak{M}} \\
& =1+\text { gl. } \operatorname{dim} S .
\end{aligned}
$$

Thus, the theorem will be proved if we prove the following

LEMMA. If $\mathfrak{M}$ is any maximal ideal of $S$, we have

$$
\text { l. gl. } \operatorname{dim} S\{x, d\} \geqq \text { l. gl. } \operatorname{dim} S_{\mathfrak{M}}\{x, \bar{d}\} \text {. }
$$

Proof of the lemma. Let us set $R=S\{x, d\}$ and $\bar{R}=S_{m}\{x, \bar{d}\}$. Let $\eta: S \rightarrow S_{\mathfrak{M}}$ denote the ring homomorphism defined by $\eta(s)=$ class of $s / 1$. Since $\bar{d} \circ \eta=\eta \circ d, \eta$ induces (see (2.2)) a ring homomorphism $\bar{\eta}: R \rightarrow \bar{R}$ such that $\bar{\eta} \mid S=\eta$.

We first prove the following two statements:

(1) $\bar{R}$ is $R$-flat as a right $R$-module (through $\bar{\eta}$ ).

(2) If $M$ is any left $\bar{R}$-module, there exists a left $R$-module $M^{\prime}$ and a left $\bar{R}$-isomorphism $M \approx \bar{R} \otimes_{R} M^{\prime}$.

The left $S_{\mathfrak{M}}$-isomorphism $\varphi: S_{\mathfrak{M}} \otimes_{S} R \rightarrow \bar{R}$ given by $\varphi\left(1 \otimes x^{i}\right)=$ $x^{i} \in \bar{R}$ satisfies $\varphi(1 \otimes f)=\bar{\eta}(f)$ for any $f \in R$. We have

$$
\varphi(1 \otimes f g)=\bar{\eta}(f g)=\bar{\eta}(f) \bar{\eta}(g)=\varphi(1 \otimes f) \bar{\eta}(g) .
$$

Thus, $\varphi$ is an isomorphism of right $R$-modules. Since $S_{\mathfrak{M}} \otimes_{S} R$ is right $R$-flat, (1) is proved. Let

$$
\bar{F}_{1} \stackrel{\lambda}{\longrightarrow} \bar{F} \stackrel{\mu}{\longrightarrow} M \longrightarrow 0
$$

be an exact sequence where $\bar{F}_{1}$ and $\bar{F}$ are $\bar{R}$-free with bases $\left\{e_{\alpha}\right\}$ and $\left\{f_{\beta}\right\}$ respectively. We then have

$$
\lambda\left(e_{\alpha}\right)=\eta\left(\frac{1}{s_{\alpha}}\right) \sum_{\beta} \frac{1}{\eta}\left(a_{\alpha \beta}\right) f_{\beta} ; a_{\alpha \beta} \in R, s_{\alpha} \in S-\mathfrak{M} .
$$

Let $\theta$ be the $\bar{R}$-automorphism of $\bar{F}_{1}$ defined by $\theta\left(e_{\alpha}\right)=\eta\left(s_{\alpha}\right) e_{\alpha}$. Let 
$\lambda^{\prime}=\lambda \circ \theta$. We then have

$$
\lambda^{\prime}\left(e_{\alpha}\right)=\sum_{\beta} \frac{1}{\eta}\left(a_{\alpha \beta}\right) f_{\beta},
$$

and the sequence

$$
\bar{F}_{1} \stackrel{\lambda^{\prime}}{\longrightarrow} \bar{F} \stackrel{\mu}{\longrightarrow} M \longrightarrow 0
$$

is exact. Let $F_{1}$ (resp. $F$ ) be the free $R$-module generated by $\left\{e_{\alpha}\right\}$ (resp. $\left\{f_{\beta}\right\}$ ) and let $\lambda^{\prime \prime}: F_{1} \rightarrow F$ be the $R$-homomorphism defined by

$$
\lambda^{\prime \prime}\left(e_{\alpha}\right)=\sum_{\beta} a_{\alpha \beta} f_{\beta} .
$$

It is easily seen that if we take $M^{\prime}=c \circ \operatorname{ker} \lambda^{\prime \prime}$, we have $M \approx \bar{R} \bigotimes_{R} M^{\prime}$. This proves(2). We now complete the proof of the lemma.

Let $M$ be any left $\bar{R}$-module and let $M^{\prime}$ be a left $R$-module such that (2) is satisfied. Let

$$
\cdots \longrightarrow X_{n} \stackrel{d_{n}}{\longrightarrow} X_{n-1} \longrightarrow \cdots \longrightarrow X_{0} \longrightarrow M^{\prime} \longrightarrow 0
$$

be a resolution of $M^{\prime}$ as a left $R$-module. Then

$$
\bar{R} \otimes_{R} X_{n} \stackrel{I \otimes d_{n}}{\longrightarrow} \bar{R} \otimes_{R} X_{n-1} \longrightarrow \cdots \longrightarrow \bar{R} \otimes_{R} X_{0} \longrightarrow M \longrightarrow 0
$$

is exact in view of (1). Since $\bar{R} \bigotimes_{R} X_{i}$ is $\bar{R}$-projective, it follows that $\left(\bar{R} \otimes_{R} X_{i}, 1 \otimes d_{i}\right)$ is an $\bar{R}$-projective resolution of $M$. In particular, we have $h d_{\bar{R}} M \leqq h d_{R} M^{\prime} \leqq \mathrm{gl}$. $\operatorname{dim} R$. Since $M$ is arbitrary, it follows that $\mathrm{gl} . \operatorname{dim} \bar{R} \leqq \mathrm{gl}$. $\operatorname{dim} R$. This proves the lemma and hence the theorem.

REMARK. Let $S=K\left[x_{1}, \cdots, x_{n}\right]$ be the polynomial ring in $n$ variables over a field $K$. It is well-known [7, Chap. III Cor. 4 to Th. 5] that Krull $\operatorname{dim} S_{\mathfrak{M}}$ is the same for all maximal ideals $\mathfrak{M}$ of $S$. Let $d$ be a $K$-derivation of $S$ given by $d\left(x_{i}\right)=f_{i}$. Then the derivation $d$ satisfies condition (2) of Theorem 2 if and only if $f_{i}, 1 \leqq i \leqq n$ are not coprime and in this case we may apply the theorem and we have gl. $\operatorname{dim} R=n+1$. This includes the special case of Theorem 1 of [6] in which $K$ is a field.

\section{REFERENCES}

1. H. Cartan, and S. Eilenberg, Homological Algebra, Princeton University Press, 1956.

2. S. Eilenberg, A. Rosenberg and D. Zelinsky, On the dimension of modules and algebras VIII, Nagoya Math. J. 12 (1957), 71-93. 
3. N. S. Gopalakrishnan, On some filtered rings, Proc. Ind. Acad. Sci. 56 (1962), 148-154.

4. S. Maclane, Homology, Springer-Verlag, 1963.

5. O. Ore, Theory of non-commutative polynomials, Ann. Math. 34 (1933), 480-508.

6. A. Roy, A note on filtered rings, (to appear in Arch. der Math.).

7. J. P. Serre, Algebre locale-multiplicites, Mimeographed notes by P. Gabriel, College de France, 1958.

8. R. Sridharan, Homology of non-commutative polynomial rings, (to appear).

PoONA UNIVERSITY

Tata Institute of Fundamental Research,

Bombay 5 and Centre for Advanced Training \& Research, UNIVERSITY OF BOMBAY 



\section{PACIFIC JOURNAL OF MATHEMATICS}

\section{EDITORS}

\author{
H. SAMELSON \\ Stanford University \\ Stanford, California \\ J. P. JANS \\ University of Washington \\ Seattle, Washington 98105
}

\author{
J. DUGUNDJI \\ University of Southern California \\ Los Angeles, California 90007 \\ RICHARD ARENS \\ University of California \\ Los Angeles, California 90024
}

\section{ASSOCIATE EDITORS}
E. F. BECKENBACH
B. H. NeumanN
F. WOLF
K. YOSIDA

\section{SUPPORTING INSTITUTIONS}

\author{
UNIVERSITY OF BRITISH COLUMBIA \\ CALIFORNIA INSTITUTE OF TECHNOLOGY \\ UNIVERSITY OF CALIFORNIA \\ MONTANA STATE UNIVERSITY \\ UNIVERSITY OF NEVADA \\ NEW MEXICO STATE UNIVERSITY \\ OREGON STATE UNIVERSITY \\ UNIVERSITY OF OREGON \\ OSAKA UNIVERSITY \\ UNIVERSITY OF SOUTHERN CALIFORNIA
}

STANFORD UNIVERSITY

UNIVERSITY OF TOKYO

UNIVERSITY OF UTAH

WASHINGTON STATE UNIVERSITY

UNIVERSITY OF WASHINGTON

AMERICAN MATHEMATICAL SOCIETY CHEVRON RESEARCH CORPORATION TRW SYSTEMS

NAVAL ORDNANCE TEST STATION 


\section{Pacific Journal of Mathematics}

\section{Vol. 19, No. 1 \\ May, 1966}

A. R. Brodsky, The existence of wave operators for nonlinear equations... 1

Gulbank D. Chakerian, Sets of constant width................... 13

Robert Ray Colby, On indecomposable modules over rings with minimum condition....................................... 23

James Robert Dorroh, Contraction semi-groups in a function space ....... 35

Victor A. Dulock and Harold V. McIntosh, On the degeneracy of the Kepler

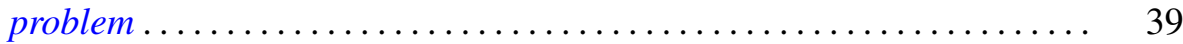

James Arthur Dyer, The inversion of a class of linear operators ......... 57

N. S. Gopalakrishnan and Ramaiyengar Sridharan, Homological dimension of Ore-extensions ................................. 67

Daniel E. Gorenstein, On a theorem of Philip Hall ................. 77

Stanley P. Gudder, Uniqueness and existence properties of bounded observables..................................... 81

Ronald Joseph Miech, An asymptotic property of the Euler function ....... 95

Peter Alexander Rejto, On the essential spectrum of the hydrogen energy and related operators ............................... 109

Duane Sather, Maximum and monotonicity properties of initial boundary

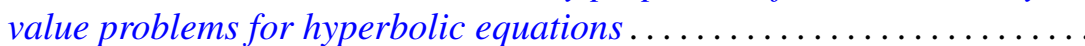

Peggy Strait, Sample function regularity for Gaussian processes with the parameter in a Hilbert space ........................... 159

Donald Reginald Traylor, Metrizability in normal Moore spaces ........... 175

Uppuluri V. Ramamohana Rao, On a stronger version of Wallis' formula ...............................

Adil Mohamed Yaqub, Some classes of ring-logics....... 